\title{
Co-Designing a Behavioural Intervention for Reducing the Impact of Chemotherapy-Induced Peripheral Neuropathy Symptoms: An Evidence- and Theory-Driven Approach
}

\author{
Mary Anne Lagmay Tanay ( $\square$ mary.tanay@kcl.ac.uk) \\ Florence Nightingale Faculty of Nursing, Midwifery and Palliative Care, King's College London \\ https://orcid.org/0000-0002-3637-6742 \\ Jo Armes
}

University of Surrey Faculty of Health and Medical Sciences

Catherine Oakley

Guy's and St Thomas' NHS Foundation Trust

\section{Liz Bryson}

Patient Expert

Robin Johnston

Patient Expert

\section{Rona Moss-Morris}

King's College London Institute of Psychiatry Psychology and Neuroscience

\section{Anne Marie Rafferty}

Florence Nightingale Faculty of Nursing, Midwifery and Palliative Care, King's College London Jose Roca

Guy's and St Thomas' NHS Foundation Trust

\section{Lesley Sage}

Patient Expert

Deb Tanner

\section{Patient Expert}

\section{Lauren Urwin}

Guy's and St Thomas' NHS Foundation Trust

\section{Toni Wyatt}

Guy's and St Thomas' NHS Foundation Trust

\section{Glenn Robert}

Florence Nightingale Faculty of Nursing, Midwifery and Palliative Care, King's College London

\section{Research Article}


Keywords: chemotherapy-induced peripheral neuropathy, cancer, chemotherapy, experience-based codesign, Common Sense Model, intervention development

Posted Date: September 29th, 2021

DOI: https://doi.org/10.21203/rs.3.rs-853039/v1

License: (1) This work is licensed under a Creative Commons Attribution 4.0 International License. Read Full License 


\section{Abstract}

\section{Purpose}

The processes and mechanisms of action which explain how behavioural interventions for chemotherapy-induced peripheral neuropathy (CIPN) work or not are unclear. We describe a co-design process for developing an evidence and theory-based behavioural intervention to reduce the impact of CIPN symptoms on patients' quality of life.

\section{Methods}

Guided by the Medical Research Council Framework for developing and evaluating complex interventions, our process of intervention development was informed by: (a) findings of systematic reviews, (b) inductive analysis of thirty-nine hours of observational fieldwork, twelve patient and eleven clinician interviews, (c) deductive analysis using the Common-Sense Self-Regulation Model and (d) fifteen patients and twenty clinicians being actively involved as co-designers.

\section{Results}

CIPN perception and coping behaviours (including, for example, self-monitoring of symptoms and engaging in self-management and safety strategies to reduce impact of CIPN symptoms) were highlighted as processes to target when co-designing an intervention. To address these behaviours, a psychoeducational approach was deemed suitable. Four behavioural change techniques informed the codesign of the intervention: (a) information about health consequences, (b) salience of consequences, (c) instruction on how to perform a behaviour and (d) action planning.

\section{Conclusion}

Current behavioural interventions for CIPN have rarely involved stakeholders, e.g. patients and clinicians, in their development process. We developed a logic model for documenting the proposed mechanism of action of our co-designed intervention for reducing impact of CIPN symptoms. This model will be refined and tested in a subsequent process evaluation as part of a feasibility trial of the intervention.

\section{Introduction}

Some neurotoxic drugs cause a condition called chemotherapy-induced peripheral neuropathy (CIPN). There is no drug recommended to prevent CIPN symptoms from developing [1] whilst Duloxetine is the only drug that shows efficacy for managing CIPN pain [2]. Alternatively, chemotherapy doses are sometimes delayed, reduced or discontinued to limit the seriousness of patients' CIPN symptoms [3].

Previous studies highlight how patients are inadequately prepared to recognise CIPN [4-7]. A perceived risk of CIPN as being lower than that of their cancer [8], perceived need to finish the complete course of chemotherapy $[8,9]$, fear of stopping treatment $[5,6,9]$ and limited understanding of long-term effects of 
CIPN [9] influence patients' reporting behaviours and their attitudes towards its management. Furthermore, patients feel inadequately supported to manage their symptoms, realising with hindsight that poorly managed CIPN symptoms negatively impact upon quality of life (QoL) [8-10].

Cognitive-behavioural interventions have been used for managing cancer symptoms such as fatigue [11] and treatment-related side-effects [12-14] including CIPN [15-17]. The literature shows benefits of cognitive-behavioural interventions to include influencing or changing cognitions, emotions and behaviours [18-20].

A systematic review of current evidence found that although behavioural interventions for CIPN exist, the processes and mechanisms of action of how they do or do not work are unclear [21]. The Medical Research Council (MRC) Framework for developing and evaluating complex interventions [22] highlights the importance of a good theoretical understanding of how an intervention causes change. This is particularly important for complex interventions with several interacting components designed to address problems shaped by the behaviours of both those who deliver and receive the intervention. Involvement of service users at all stages of the development process is also viewed as essential for making it more likely that the intervention can be successfully implemented in practice [22]. However, current behavioural interventions for CIPN have rarely - if at all - involved stakeholders e.g. patients (recipients of the intervention) and clinicians (who deliver the interventions) in their development process [21].

This study aims to co-design an evidence and theory-based behavioural intervention to reduce the impact of CIPN symptoms on patients' quality of life. We (a) describe an evidence and theory-based approach to co-designing complex interventions; (b) share our experience of using this approach; (c) present a selfregulation model of CIPN and (d) propose a behavioural intervention for reducing the impact of CIPN symptoms. We use the Guidance for reporting intervention development studies in health research (GUIDED) [23] to structure our findings, and the template for intervention description and replication (TIDieR) checklist [24] to report and describe the intervention as recommended by GUIDED.

\section{Methods}

The study was guided by the MRC Framework for developing and evaluating complex interventions [22]. Building upon a thematic synthesis of CIPN patient experience [8] and systematic review of behavioural interventions in CIPN management [21] previously conducted by the lead author, we employed Experience-Based Co-Design (EBCD) [25] as a collaborative process to systematically develop the complex intervention with patients and clinicians. This process was informed theoretically by the Common-Sense Model of Self-Regulation [26].

(1) Intervention development using the EBCD approach

Stage 1: Setting up the project 
The study was conducted in oncology clinics and a chemotherapy unit at a London cancer centre. Senior clinicians and managers were involved in planning study processes and arranging access to participants. Two patients who had experienced CIPN reviewed the study protocol and ethics applications (referred to as the non-clinical CIPN expert group or NCEG).

Following ethical and site approval, convenience purposive sampling was used to approach potential patient participants. Adults diagnosed with either breast or colorectal cancer, and who were due to receive or who were actively receiving neurotoxic chemotherapy were invited to participate. Staff involved in obtaining consent and/or conducting chemotherapy-related consultations with patients were recruited. All participants signed written informed consent and had the option to attend one or more of the study activities i.e. interview, observation, and workshops (see below).

\section{Stage 2 and 3: Engaging patients and staff and gathering their experiences}

Experience data were collected through observations of chemotherapy patient-clinician consultations, clinician interviews and filmed/audio recorded patient interviews. Inductive analysis of interview and observation data was carried out. Findings have been published elsewhere (cite qual paper here).

Patient interviews were analysed thematically, edited and compiled by MT into a 'trigger film'. This film explored key themes and issues based on the experiences of people [27] who are at risk of or already have CIPN symptoms. Fundamental to the co-design process, key 'emotional touchpoints' in patients' experiences were highlighted in the trigger film [28].

\section{Patient feedback workshop}

The workshop was attended by members of the NCEG and study patient participants. Patients who were unable to attend were invited to send their comments by email. The trigger film was shown for patients to check for accuracy and resonance with their experiences, as well as to encourage further discussion (member checking) [29]. An emotional mapping exercise was facilitated by MT where patients shared how they felt as recipients of information and support for CIPN [30,31] at different points of their treatment journey. Following discussions, participants identified key patient priorities for improvement.

\section{Clinician feedback workshop}

Thematic analysis of findings from clinician interviews were presented to clinicians, followed by discussion of the broad priorities identified in the interviews and a prioritising exercise. Participants were asked to hypothetically allocate proportions of a set amount of funds according to what they perceived should be prioritised. Further discussions resulted in a set of agreed key clinician priorities.

\section{Stage 4: Joint workshop (co-design)}

Clinician and patient group priorities were presented by a nominated representative from each group. The trigger film was then shown; the first instance for clinicians to view the film. 
In three mixed patient-clinician groups, participants discussed the issues presented in the film and group priorities. Each mixed group was given printed materials describing patient and clinician priorities and was asked to discuss and then reprioritise these. They then shared their views with the larger group to agree on a final set of joint priorities.

\section{Stage 5: Small co-design teams}

Specific tasks essential to intervention development were divided among smaller co-design teams. All participants were grouped into these patient-clinician teams to work on specific tasks in an iterative design and development process. Graphic designers and a film editor who provided creative input.

\section{Stage 6: Celebration and progression event}

Ten months after the initial joint event, a celebration event was held. This was co-presented by nominated patient group representatives and clinician group representatives. The purpose of the event was to provide the study update, present the co-designed intervention, reflect upon the co-design process and inform participants of the next steps. The event was attended by patient and clinician participants, NCEG and other invited stakeholders who were not directly involved in the co-design process.

All workshops were audio-recorded and co-facilitated by MT, who has extensive facilitation experience in higher education settings. Researcher field notes and workshop transcriptions were analysed, then communicated back to the co-design teams to inform the focus of both the subsequent workshop and ongoing intervention development.

(2) Theoretical modelling using the Common-Sense Model

The use of existing theory when developing interventions is proposed within the MRC Framework [22]. During initial inductive analysis of workshop discussions and comparisons with earlier interview and observation findings, the core constructs from the Common-Sense Model of Self-Regulation (CSM) [26, 32] were recognised as relating to the discussions and outputs. CSM focuses on how individual cognitive and emotional illness representations influence action plans and behaviours for self-management [26]. Although CSM is largely used to understand perception of illness e.g. cancer, it has been used in research to understand perceptions of key symptoms such as fatigue [33] and pain [34]. CSM research has identified six cognitive illness-representations dimensions namely: identity (beliefs about how the condition is identified or labelled and symptoms associated with the label); timeline (beliefs related to how long the illness might last); consequences (beliefs about the physical and social effects of the specific health condition on them); cause (perceived reason or cause for the development of the illness); controllability (the individual's beliefs of how much they or their treatment can manage or control the illness and its symptoms) and illness coherence (whether the person feels they have a coherent understanding of the illness) $[26,35]$

Following the first joint and two succeeding workshops with smaller co-design teams, a separate online workshop was attended by patients in which participants validated the key dimensions of CSM with their 
CIPN experiences. Using CSM as a framework, MT deductively analysed the qualitative data and workshop discussions to understand perceptions of and coping strategies for managing CIPN. Themes were reviewed by GR and JA, followed by confirmation and sense-checking by patient participants. Our emerging analysis informed ongoing intervention modelling by the smaller co-design teams.

(3) Developing a logic model

The MRC Framework recommends a clear description of the intervention and its causal assumptions be provided [22]. We developed a logic model [36] to diagrammatically present a description of the anticipated intervention components (the intended activities), delivery mechanisms (the structures in place to deliver the intervention), mechanisms of impact (our assumptions about how the intervention will produce intended effects) and intended outcomes. Behaviour change techniques (BCT) were specified using the Behaviour Change Taxonomy version 1[37] and links between BCTs and mechanisms of action (MoA) were identified using information from a guide developed from a synthesis of BCT-MoA links as frequently described in the literature [38].

Intervention development was completed within 15 months (longer than initially planned due to the COVID-19 pandemic). Figure 1 provides an overview of the key elements of the intervention co-design process.

\section{Results}

\section{Participants}

All participants were given the option of attending one or more study activities. In total, there were 42 EBCD participants across all stages. Five patient participants and five clinicians participated in all stages. Four members of the NCEG attended all workshops. Due to COVID-19 government restrictions, only two initial small co-design workshops were conducted face-to-face; all subsequent workshops $(n=7)$ were conducted virtually. Follow-up emails were exchanged between members of the co-design teams after workshops.

Table 1 shows involvement according to type of participants and by EBCD stage.

\section{Identified priorities}

Following the separate patient and clinician workshops, priorities for improving patient experience of CIPN were identified by each group. Both groups identified issues in relation to CIPN information provision and the lack of awareness of available resources and opportunities for managing CIPN. Using the SMART criteria (specific, measurable, attainable, relevant and time-bound) [39], co-design team members agreed joint priorities. A broad description of an initial intervention prototype was conceived following discussions. Smaller co-design teams addressed four workstreams: (a) mapping of available 
information and support for managing CIPN, (b) structuring and collating components of patient information booklet and (c) planning components of film narrative and timing of delivery and (d) working with graphic designers and film editor for booklet layout and film composition. A summary of patient, clinician and agreed priorities and workstreams are shown on Table 2.

\section{A self-regulation model of CIPN}

The Common-Sense Model (CSM) of Self-Regulation enhanced our understanding of the complex processes involved in experiences of CIPN and ways to address this condition.

\section{Societal factors}

Societal factors that contribute to the interpretative process through which the individual formulates their perception of CIPN were highlighted. How individuals coped with CIPN was influenced by the roles they carry out e.g. at home or in the workplace; and what they perceived to be a general societal lack of awareness of CIPN. Early cognitive and emotional representations of CIPN were influenced by the attitudes of clinicians and the information and descriptions given by them, which coincided with information-overload about the acute and potentially life-threatening side-effects of cancer treatments. Individuals were less in favour of reducing chemotherapy dose for controlling symptoms.

\section{Cognitive representations of CIPN}

Attributes of the cognitive representation of CIPN include causal beliefs about symptoms (that influence how the individual labels symptoms and creates identity for CIPN); perceived inevitability of CIPN due to cancer treatment; and perceived ability to control the symptoms (which is affected by the individuals' perceived timeline of symptoms). Patient's perceived understanding of CIPN symptoms increased when symptoms were experienced and when symptoms affected activities involving their hands or feet. When symptoms become more noticeable and begin to cause problems, patients also report their symptoms.

\section{Emotional representations of CIPN}

The threatening situation, combined with the cognitive beliefs, can trigger emotional distress and low mood, prompting emotion-focused coping such as minimisation of the problem (not reporting how severe their symptoms were) or avoidance (not reporting their symptoms or avoiding conversations about CIPN). Patients' perception of CIPN was also influenced by fear of death from cancer.

The most common coping strategies individuals used were information-seeking, positive reframing, seeking social support, active coping and acceptance of the symptoms. However, reappraisal of coping strategies was not observed during their treatment. Rather, patients viewed CIPN as part of an acceptable new norma/ within the context of cancer effects and treatment side-effects.

Figure 2 illustrates a self-regulation model to conceptualise CIPN. Exemplars of participant quotes are provided in Supplementary Table 1. 


\section{The REACT-CIPN intervention}

Guided by the CSM, the processes to be targeted in our intervention are CIPN perception and coping behaviours namely, (a) self-monitoring of symptoms, (b) communicating and early reporting of symptoms to clinicians, (c) participating in making chemotherapy dose reduction decisions with their clinicians, and (d) engaging in self-management and safety strategies to reduce impact of CIPN symptoms. We identified potential facilitators of these behaviours and mapped these on to CSM constructs that influence CIPN symptom perception (Table 3). To address these behaviours, a psychoeducational approach i.e. booklet and film was deemed suitable. The intervention name agreed by the co-design team was REACT-CIPN (ㄹeducing the Impact of Chemotherapy Induced Peripheral Neuropathy). The REACT-CIPN booklet and film are complementary in targeting behavioural processes.

Behavioural change techniques (BCTs) used are (a) information about health consequences, (b) salience of consequences, (c) instruction on how to perform a behaviour and (d) action planning. Proposed BCTs were guided by our findings from the literature reviews [8, 21], qualitative data analyses and co-design workshops. For example, boosting perceived CIPN identity by providing information about acute and long-term CIPN symptoms, CIPN incidence, and hints on how to recognise early symptoms and when to report these. Visual representations of CIPN symptoms and impact on common daily tasks were also included in the booklet and film. Providing information about adaptive coping safety strategies or selfmanagement and available expert support can enhance perceived control (self) and control (treatment), respectively.

Instructions for using the intervention will be given by the clinician before commencing chemotherapy treatment (booklet) and after chemotherapy cycle 2 (film). Details of the REACT-CIPN intervention components, delivery mechanisms, BCTs [37] and their mechanisms of action [38], and outcomes are illustrated on Figure 3.

\section{Discussion}

When developing complex interventions, a dynamic, systematic and iterative approach is necessary for illuminating complex processes $[22,40]$. In our experience, the combination of a theory- and evidencebased co-design approach was particularly valuable. The MRC Framework [22] offers a transparent, methodical and coherent approach to address health needs using research evidence, theory and experiences of patients and clinicians to co-design a tailored intervention that targets behaviours in a specific patient group. There are several characteristics of our approach that future intervention developers may wish to consider.

The first is the robust evaluation of published research evidence and user experience to underpin the development of an intervention. From the initial phases of intervention development, our theory and evidence-based approaches enabled us to: (a) gain detailed insight into the context of CIPN experience and patients' needs [8] (cite qual paper here), (b) identify mechanisms of action of current behavioural interventions for reducing CIPN symptoms and potential barriers to intervention success [21] and (c) 
incorporate existing knowledge into our intervention. Previous research shows that illness perceptions can act as mediators between illness activity and coping styles [41]. We have drawn on the CSM to interpret empirical determinants of CIPN perceptions into theoretical constructs. To our knowledge, no research has applied the self-regulation model to understand the subjective experiences of patients with CIPN.

In our study, several social responses to CIPN symptoms were shown to have influenced cognitive and emotional representations. For example, the lack of societal CIPN awareness implies that for many patients, their initial perception of CIPN formed when they were told (for the first time) by their clinicians about all potential side-effects of their chemotherapy treatment. CIPN information provided by clinicians is key to developing CIPN perceptions, thus timing must be considered. Strategies such as reinforcement of CIPN information at cycle 2 onwards, availability of information in formats preferred by patients, and easily accessible CIPN information were suggested.

Aside from recognising the symptoms and impact (identity) of CIPN, the patient's perception of CIPN will influence interpretation of the cause of the symptoms. This is particularly challenging because the threat of cancer is clearly more important than eliminating the cause of CIPN i.e. the chemotherapy that could save their lives. The consequences of continuing or stopping chemotherapy will be internalised and made part of the person's CIPN representation and resulting behaviours. Further, perceived control of CIPN symptoms will influence reporting or self-managing behaviours which can affect symptom severity.

In addition, a person's ability to change the overall timeline or duration of symptoms, with respect to reporting or making chemotherapy dose reduction decisions and self-management, will be an important component of the person's CIPN representation. The ongoing interaction between internal (i.e., impact of CIPN on quality of life) and external factors (i.e. clinician and public opinion) changes the illness representation over time and further guides the individual's actions in response to the threat (CIPN) [26, 41]. Emotions (e.g. fear, anger, distress, low mood, avoidance) in CIPN also contribute towards formation of illness representation and develop together with the cognitive component [26, 41]. These emotions can motivate the individual to create an action plan or coping strategy. On the other hand, they can be overwhelming and lead to limited, or lack of action or coping. This theoretical understanding of the CIPN experience helped us to recognise target behavioural processes and select appropriate behaviour change techniques aiming to promote target behaviours.

Complementing theory and evidence-based approaches, the EBCD approach ensured patient needs and perspectives were understood and clinicians' perspectives were accommodated to maximise engagement and future implementation. Although EBCD has been used predominantly for quality improvement [42], this was not the first instance of this approach being used in developing complex interventions in cancer care [43]. Co-design approaches encourage patients to impart their perspective and voice and allow them to meaningfully reflect upon their shared experiences with their clinicians. Instead of being passive sources of information, patients become active partners in prompting change [25]. Realignment of power dynamics between service users (patients) and service providers (clinicians) and sustained involvement 
of each as partners in a change process are key to successful co-design in healthcare [42]. Whilst we faced several challenges - including restrictions due to the COVID-19 pandemic - we were able to maintain momentum and retained sufficient patients and clinicians in all six stages of EBCD. The flexible nature and level of involvement in various stages of the EBCD process maintained involvement and reduced attrition. We also emphasise the role of the researcher as facilitator and mediator in maintaining momentum. The importance of facilitation in co-design stages has been highlighted in the past, particularly when joint priorities are set and co-design activities are planned [42].

We acknowledge limitations of this study. Firstly, combining purposive sampling and the option to engage in chosen study activities may have resulted in a sample that was not representative of the wider population of patients with CIPN. Despite efforts to include interview participants from ethnic minority backgrounds in the co-design activities, no participants from this group of interviewees consented to participate. This is a common observation in healthcare studies including participatory health research [44]. To address this, the researchers ensured that data from their qualitative interviews (cite qual paper here) were considered by the co-design teams. Patient participants in the co-design teams who reviewed intervention information ensured these were presented in plain English and that no medical jargon was used. Visual images showing hands and feet were inclusive and portrayed diverse races and genders. Secondly, patients frustrated with CIPN-related issues might have been more likely to participate in the study and all activities. Conversely, clinicians who were particularly interested in the topic and so were motivated to improve CIPN practice and services were more likely to join. The collaborative and usercentred nature of the EBCD approach enabled participants to reflect on their shared experiences, to identify issues and to explore potential solutions together. Finally, the first author of this paper analysed the data and was also the facilitator in the workshops. Previous experiences, personal beliefs and objectives and cultural background of the researcher could bias analysis and reporting [45]. However, efforts were made to minimise this bias by continuous sense checking with the participants. Additionally, representative four patient and four clinician members of the co-design teams were involved in coauthoring this article.

\section{Conclusion}

In co-designing this intervention, important behavioural change techniques which informed its development were (a) information about health consequences, (b) salience of consequences, (c) instruction on how to perform a behaviour and (d) action planning. We developed a logic model vital for documenting the proposed mechanisms of action of the co-designed intervention for reducing impact of CIPN symptoms. This model will be refined and tested in the process evaluation phase of a subsequent feasibility trial of the intervention.

\section{Declarations}

\section{Funding}


This paper presents independent research funded by the National Institute for Health Research (NIHR Doctoral Research Fellowship, Mary Anne L. Tanay DRF-2018-11-ST2-017). The views expressed are those of the authors and not necessarily those of the NHS, the NIHR or the Department of Health and Social Care.

\section{Conflict of interest/Competing interest}

The authors declare that there is no conflict of interest.

\section{Availability of data and material}

The datasets during and/or analysed during the current study are available from the corresponding author on reasonable request. The authors have full control of all primary data which is available upon request.

Code availability: Not applicable

\section{Authors' contributions}

MT conceived and designed the study, facilitated the co-design workshops, qualitatively analysed the data, drafted the manuscript and led the coordination of the study. GR, JA, RMM and AMR contributed to the design, participated in data analysis and helped draft the manuscript. CO, JR, TW, LU, LB, RJ, LS and DT participated in co-design workshops and helped draft the manuscript. All authors read and approved the final manuscript.

\section{Ethics approval}

East Midlands - Leicester South Research Ethics Committee REC (Reference 19/EM/0192) assessed and approved the study.

\section{Consent to participate}

All study participants gave consent and signed a consent form.

Consent for publication: Not applicable

\section{Acknowledgments}

We are indebted to all the patients and clinicians who participated in this research and made it possible. MT and GR are affiliated to the Samskapa research programme on co-production led by Jönköping University which helped inform the conduct and analysis of this study. This research programme is funded by Forte, the Swedish Research Council for Health, Working Life and Welfare under grant agreement no. 2018-01431. 


\section{References}

1. Jordan, B., et al., Systemic anticancer therapy-induced peripheral and central neurotoxicity: ESMO\&\#x2013;EONS\&\#x2013;EANO Clinical Practice Guidelines for diagnosis, prevention, treatment and follow-up. Annals of Oncology, 2020. 31(10): p. 1306-1319.

2. Loprinzi, C.L., et al., Prevention and Management of Chemotherapy-Induced Peripheral Neuropathy in Survivors of Adult Cancers: ASCO Guideline Update. J Clin Oncol, 2020. 38(28): p. 3325-3348.

3. Park, S.B., et al., Chemotherapy-induced peripheral neurotoxicity: a critical analysis. CA Cancer J Clin, 2013. 63(6): p. 419-37.

4. Tofthagen, C., Surviving chemotherapy for colon cancer and living with the consequences. J Palliat Med, 2010. 13(11): p. 1389-91.

5. Tofthagen, C., Patient perceptions associated with chemotherapy-induced peripheral neuropathy. Clin J Oncol Nurs, 2010. 14(3): p. E22-8.

6. Bakitas, M.A., Background noise: the experience of chemotherapy-induced peripheral neuropathy. Nurs Res, 2007. 56(5): p. 323-31.

7. Boehmke, M.M. and S.S. Dickerson, Symptom, symptom experiences, and symptom distress encountered by women with breast cancer undergoing current treatment modalities. Cancer Nurs, 2005. 28(5): p. 382-9.

8. Tanay, M.A.L., J. Armes, and E. Ream, The experience of chemotherapy-induced peripheral neuropathy in adult cancer patients: a qualitative thematic synthesis. Eur J Cancer Care (Engl), 2017. 26(5).

9. Salgado, T.M., et al., Reporting of paclitaxel-induced peripheral neuropathy symptoms to clinicians among women with breast cancer: a qualitative study. Support Care Cancer, 2020. 28(9): p. 41634172.

10. Tanay, M.A. and J. Armes, Lived experiences and support needs of women who developed chemotherapy-induced peripheral neuropathy following treatment for breast and ovarian cancer. Eur J Cancer Care (Engl), 2019: p. e13011.

11. Corbett, T.K., et al., The effectiveness of psychological interventions for fatigue in cancer survivors: systematic review of randomised controlled trials. Systematic reviews, 2019. 8(1): p. 324-324.

12. Hunter, J.J., et al., A randomized trial of nurse-administered behavioral interventions to manage anticipatory nausea and vomiting in chemotherapy. Cancer Med, 2020. 9(5): p. 1733-1740.

13. Given, C.W., et al., Managing Symptoms Among Patients With Breast Cancer During Chemotherapy: Results of a Two-Arm Behavioral Trial. Journal of Clinical Oncology, 2008. 26(36): p. 5855-5862.

14. Berry, D.L., et al., Electronic Self-Report Assessment for Cancer and Self-Care Support: Results of a Multicenter Randomized Trial. Journal of Clinical Oncology, 2014. 32(3): p. 9.

15. Knoerl, R., et al., Self-Guided Online Cognitive Behavioral Strategies for Chemotherapy-Induced Peripheral Neuropathy: A Multicenter, Pilot, Randomized, Wait-List Controlled Trial. The Journal of Pain, 2018. 19(4): p. 382-394. 
16. Knoerl, R., et al., Exploring the efficacy of an electronic symptom assessment and self-care intervention to preserve physical function in individuals receiving neurotoxic chemotherapy. Biomed Central Cancer, 2019. 18(1203): p. 1-13.

17. Tofthagen, C., et al., Tofthagen C, Kip KE, Passmore D, Loy I, Berry DL. Usability and Acceptability of a Web-Based Program for Chemotherapy-Induced Peripheral Neuropathy. Computers Informtics Nursing, 2016. 34(7): p. 8.

18. Newell, S.A., R.W. Sanson-Fisher, and N.J. Savolainen, Systematic review of psychological therapies for cancer patients: overview and recommendations for future research. J Natl Cancer Inst, 2002. 94(8): p. 558-84.

19. Daniels, S., Cognitive Behavior Therapy for Patients With Cancer. Journal of the advanced practitioner in oncology, 2015. 6(1): p. 54-56.

20. Redd, W.H., G.H. Montgomery, and K.N. DuHamel, Behavioral Intervention for Cancer Treatment Side Effects. JNCl: Journal of the National Cancer Institute, 2001. 93(11): p. 810-823.

21. Tanay, M.A.L., et al., A systematic review of behavioural and exercise interventions for the prevention and management of chemotherapy-induced peripheral neuropathy symptoms. Journal of Cancer Survivorship, 2021.

22. Craig, P., et al., Developing and evaluating complex interventions: the new Medical Research Council guidance. BMJ, 2008. 337: p. a1655.

23. Duncan, E., et al., Guidance for reporting intervention development studies in health research (GUIDED): an evidence-based consensus study. BMJ Open, 2020. 10(4): p. e033516.

24. Hoffmann, T.C., et al., Better reporting of interventions: template for intervention description and replication (TIDieR) checklist and guide. BMJ : British Medical Journal, 2014. 348: p. g1687.

25. Robert, G., et al., Patients and staff as codesigners of healthcare services. BMJ Open, 2015.

26. Leventhal, H., L.A. Phillips, and E. Burns, The Common-Sense Model of Self-Regulation (CSM): a dynamic framework for understanding illness self-management. J Behav Med, 2016. 39(6): p. 935946.

27. Tsianakas, V., et al., Using patients' experiences to identify priorities for quality improvement in breast cancer care: patient narratives, surveys or both? BMC Health Services Research, 2012. 12(271).

28. Papoulias, C., Showing the Unsayable: Participatory Visual Approaches and the Constitution of 'Patient Experience' in Healthcare Quality Improvement. Health Care Anal, 2018. 26(2): p. 171-188.

29. Birt, L., et al., Member Checking:A Tool to Enhance Trustworthiness or Merely a Nod to Validation? Qualitative Health Research, 2016. 26(13): p. 1802-1811.

30. Donetto, S., et al., Experience-based Co-design and Healthcare Improvement: Realizing Participatory Design in the Public Sector. The Design Journal, 2015. 18(2): p. 227-248.

31. Donetto, S., Tsianakas, V. \& Robert, G., Using Experience-based Co-design to improve the quality of healthcare: mapping where we are now and establishing future directions: Final Report. 2014, King's College London: London. 
32. McAndrew, L.M., et al., Using the common sense model to design interventions for the prevention and management of chronic illness threats: from description to process. $\mathrm{Br} \mathrm{J}$ Health Psychol, 2008. 13(Pt 2): p. 195-204.

33. Corbett, T., et al., Cancer-related fatigue in post-treatment cancer survivors: application of the common sense model of illness representations. BMC Cancer, 2016. 16(1): p. 919.

34. Bunzli, S., et al., Making Sense of Low Back Pain and Pain-Related Fear. J Orthop Sports Phys Ther, 2017. 47(9): p. 628-636.

35. Moss-Morris, R., et al., The revised illness perception questionnaire (IPQ-R). Psychology and health, 2002. 17(1): p. 1-16.

36. Kellogg Foundation, W.K., Logic model development guide. 2004, Battle Creek, MI: W.K Kellogg Foundation.

37. Michie, S., et al., The behavior change technique taxonomy (v1) of 93 hierarchically clustered techniques: building an international consensus for the reporting of behavior change interventions. Ann Behav Med, 2013. 46(1): p. 81-95.

38. Carey, R.N., et al., Behavior Change Techniques and Their Mechanisms of Action: A Synthesis of Links Described in Published Intervention Literature. Ann Behav Med, 2019. 53(8): p. 693-707.

39. Blaine Lawlor, K., Smart Goals: How the Application of Smart Goals can Contribute to Achievement of Student Learning Outcomes. Developments in Business Simulation and Experiential Learning, 2012.

39: p. 259-267.

40. O'Cathain, A., et al., Guidance on how to develop complex interventions to improve health and healthcare. BMJ Open, 2019. 9(8): p. e029954.

41. Diefenbach, M.A. and H. Leventhal, The Common-Sense Model of Illness Representation: Theoretical and Practical Considerations. Journal of Social Distress and Homelessness, 1996. 5(1): p. 11-38.

42. Green, T., et al., Use and reporting of experience-based codesign studies in the healthcare setting: a systematic review. BMJ Qual Saf, 2020. 29(1): p. 64-76.

43. Tsianakas, V., et al., Enhancing the experience of carers in the chemotherapy outpatient setting: an exploratory randomised controlled trial to test impact, acceptability and feasibility of a complex intervention co-designed by carers and staff. Support Care Cancer, 2015. 23(10): p. 3069-80.

44. Roura, M., et al., Participatory health research with migrants: Opportunities, challenges, and way forwards. Health expectations : an international journal of public participation in health care and health policy, 2021. 24(2): p. 188-197.

45. Green, J. and N. Thorogood, Qualitative Methods for Health Research. 4th ed. 2018, London: SAGE.

\section{Tables}

Table 1. Types of participants and activities of EBCD stages in this study 


\begin{tabular}{|c|c|c|}
\hline Stage & $\begin{array}{l}\text { Type of } \\
\text { participants }\end{array}$ & Activities \\
\hline $\begin{array}{l}\text { EBCD Stage } \\
1: \\
\text { Setting up }\end{array}$ & $\begin{array}{l}\text { Researcher } \\
\text { (MT) }\end{array}$ & $\begin{array}{l}\text { - Ethics approval. } \\
\text { - Engagement with non-clinical CIPN expert group (NCEG) and } \\
\text { clinician collaborators. } \\
\text { - Meetings with senior clinicians and managers. }\end{array}$ \\
\hline $\begin{array}{l}\text { EBCD Stages } \\
2 \text { and 3: } \\
\text { Interviews }\end{array}$ & $\begin{array}{l}\text { Patients }(n=11) \\
\text { Clinicians }(n=8) \\
\text { Group interview } \\
\text { with clinicians } \\
(n=4)\end{array}$ & $\begin{array}{l}\text { - Semi-structured qualitative interviews. } \\
\text { - Patient interviews were filmed, analysed and edited by MT } \\
\text { into a } 20 \text {-minute film (trigger film). }\end{array}$ \\
\hline $\begin{array}{l}\text { EBCD Stages } \\
2 \text { and 3: } \\
\text { Observations }\end{array}$ & $\begin{array}{l}\text { Patients }(n=9) \\
\text { Clinicians }(n=8)\end{array}$ & $\begin{array}{l}\text { - Non-participant observations were undertaken in colorectal } \\
\text { and breast cancer clinics and at clinician stations, including the } \\
\text { observation of chemotherapy consultations between patients } \\
\text { and clinicians. }\end{array}$ \\
\hline $\begin{array}{l}\text { EBCD Stages } \\
2 \text { and 3: } \\
\text { Patient } \\
\text { feedback } \\
\text { workshop }\end{array}$ & $\begin{array}{l}\text { Patients }(n=5) \\
\text { NCEG }(n=3)\end{array}$ & $\begin{array}{l}\text { - Group updated about study progress in line with EBCD } \\
\text { stages. } \\
\text { - Trigger film was shown to participants. } \\
\text { - Emotional mapping exercise to identify emotional } \\
\text { touchpoints during their chemotherapy journey. } \\
\text { - Discussions to identify priorities for improving patient } \\
\text { experience and attributes of the intervention. } \\
\text { - Workshop was facilitated by MT and LS. }\end{array}$ \\
\hline $\begin{array}{l}\text { EBCD Stages } \\
2 \text { and 3: } \\
\text { Clinician } \\
\text { feedback } \\
\text { workshop }\end{array}$ & Clinicians $(n=5)$ & $\begin{array}{l}\text { - Group updated about study progress in line with EBCD } \\
\text { stages. } \\
\text { - Summary of qualitative interview findings were presented by } \\
\text { CO. } \\
\text { - Discussions to identify priorities for improving patient } \\
\text { experience and attributes of the intervention. } \\
\text { - Prioritising exercise using a hypothetical fund (£100) to } \\
\text { allocate on priority items. } \\
\text { - Made decisions about key priorities. } \\
\text { - Workshop was facilitated by MT. }\end{array}$ \\
\hline $\begin{array}{l}\text { EBCD Stage } \\
\text { 4: } \\
\text { Joint } \\
\text { workshop (co- } \\
\text { design) }\end{array}$ & $\begin{array}{l}\text { Patients }(n=5) \\
\text { NCEG }(n=3) \\
\text { Clinicians }(n=8)\end{array}$ & $\begin{array}{l}\text { - Group updated about study progress in line with EBCD } \\
\text { stages. } \\
\text { - Trigger film was shown to participants (first time seen by } \\
\text { clinicians). }\end{array}$ \\
\hline
\end{tabular}


- Patient priorities were presented by RJ and another patient participant.

- Clinician priorities were presented by JR and another colleague.

- Workshop was facilitated by MT.

EBCD Stage Patients $(n=5) \quad$ - Smaller co-design groups met for a total of 7 workshops.

5:

NCEG $(n=4)$

Small co-

Clinicians $(n=6)$

- One workshop was with patients, NCEG and a visual

design

workshops

illustrator to co-design visual illustrations of CIPN.

- Clinicians reviewed and provided opinion on the content and visual illustrations. Ensuing discussions between patients, NCEG and clinicians were through email (due to pandemic restrictions).

- Workshops were facilitated by MT.

EBCD Stage

6: Celebration

Event
Patients $(n=3)$

NCEG $(n=4)$

Clinicians $(n=8)$

Graphic designers $(n=1)$

Other invited clinicians [not part of $\mathrm{CO}^{-}$ design team]: $(\mathrm{n}=3)$

Researchers $(n=2)$
- Presentation of the intervention by DT.

- LS and RJ, on behalf of patients, shared their experiences of being part of the intervention development process.

- LU and TW, on behalf of clinicians, shared their experiences of being part of the study.

- Group discussion at the end of presentations.

- Closed with announcement of next phase.

- Event was facilitated by MT.

Table 2. Summary of EBCD priorities and workstreams 


$\begin{array}{lll}\text { Patient- } & \text { Patients do not } & \text { Improve ways of } \\ \text { identified } & \text { retain CIPN } & \text { providing helpful CIPN } \\ \text { priorities } & \text { information. } & \text { patient information }\end{array}$

\section{Content:}

- Explains CIPN in words that describe the symptoms well - use examples of patient experiences; broaden the language not just tingling, spell out the various forms of CIPN and how symptoms affect an individual

- Gives clear information of the differences between acute and longterm CIPN symptoms and impact

- Provides clarity about temporality versus permanence of symptoms

- Highlights how symptoms may affect individual hobbies, daily activities and career/livelihood

- Suggests practical tips for managing symptoms

- Provides information about CIPN support available and who to contact

- Gives balanced information about risk of developing CIPN and completing treatment

- Encourages patients to participate in making decisions about reducing or stopping treatment

- Provides guidance for early reporting of CIPN symptoms and how to communicate these to clinicians

- Provides reminders about CIPN symptoms and what to watch out for throughout the chemotherapy treatment

- Includes voices of persons who have experienced CIPN

\section{Design and layout of written information/leaflet:}

- Highlights key information succinctly and visually

- Uses visual representation of incidence to help inform likelihood of 
- Provides easily accessible format, depending on patient's preference, such as hard copy booklet or online access

- Allows space for recording questions patients may have for their clinicians about CIPN

Patients' perceived lack of awareness about CIPN among carers, friends and employers.
Provide CIPN information for carers, families and employers.
- Provides resources that can be shared with carers, friends and employers to raise awareness without exaggerating
Patients' perceived lack of support for managing CIPN after treatment.

Patients' perceived lack of CIPN knowledge and understanding, particularly symptommanagement, among clinicians.
Consider support available for managing CIPN after treatment.

Provide CIPN education for clinicians, including GPs.
- Provides group classes or support groups for CIPN
- Ensures patient involvement when developing patient information material
Clinician identified priorities
No standard CIPN information provided to patients.
Improve ways of providing CIPN patient information.

- Develop patient information resources that are consistent and in multiple formats

- Provide information about CIPN which should include risk, nature of CIPN symptoms, prevention and management

- Identify the best time to provide CIPN information

- Encourage patient-led monitoring and reporting

- Provide information which should cover all stages from prechemotherapy until follow-up and what to report in every stage

Need for education and training for clinicians about CIPN.
Provide learning opportunities for clinicians about CIPN.
- Develop evidence-based CIPN protocol/guidance

- Develop a video or film about CIPN and how to prevent or manage symptoms 
of support for managing CIPN provided by other departments. with members of the MDT involved.

clinicians

- Develop strategy for early referral to CIPN rehabilitation services

- Provide clinicians reminders about available CIPN support and services (for regular and new staff)

- Expand service capabilities

\section{Agreed joint priorities guided by SMART criteria $[39,40]$}

To develop patientinformed information materials for patients and also aid clinicians who give information about CIPN to patients.

\section{Co-design workstreams}

- Workstream 1: Mapping of available information and support for managing CIPN. Results will inform the patient information materials.

- Workstream 2: Structuring and collating components of patient information booklet including method/s of delivery.

- Workstream 3: Planning components of film narrative and timing of delivery.

- Workstream 4: Working with graphic designers for booklet layout and film composition.

Table 3. Proposed target behaviours for managing CIPN symptoms and facilitators 
Target behaviours

Self-monitoring of symptoms

\section{Facilitators}

Patients are more likely to perform behaviour if they:

[Evidence: a. thematic synthesis of CIPN patient experience [8], b. systematic review of CIPN behavioural interventions [21], c. interviews and observations, d. codesign activities]

- are encouraged to assess the importance of

maintaining function of their hands and feet in relation to their livelihood, domestic roles and hobbies and how CIPN symptoms may affect these. (c, d)

illness

coherence

consequences

- have understanding and knowledge about CIPN e.g. identity acute and long-term CIPN symptoms and incidence, duration of symptoms, and reason for the development timeline of CIPN. $(a, b, c, d)$

- are able to envision CIPN symptoms and potential impact on common daily tasks through patientinformed visual representations. (a, c, d)

cause

- are provided with information that is easily accessible when needed. (b, d)

identity

consequences

identity

consequences symptoms to clinicians
Communicating and early reporting of

- are provided with information about how to recognise early symptoms, when to report and consequences of not reporting. (b, c, d)

- are encouraged to self- monitor symptoms and communicate their symptoms to their clinicians e.g. note-taking, what information to mention. (b, c, d)

- are given reinforcement and reminders i.e. information given at different timepoints of chemotherapy treatment. (c, d)

- are encouraged to participate in decision-making concerning their treatment. (a, c, d)

- are provided with instructions on how to adapt selfmanagement and safety strategies. (b, c, d)

Engaging in selfmanagement and safety strategies to reduce impact of CIPN symptoms
- have increased awareness of available expert support and treatments for managing CIPN symptoms and reducing impact. (b, c, d)

- are empowered to share information materials with their families, carers, employers and other clinicians such as their GP. (c, d)

- are motivated to understand and gain control of their own symptoms and engage in self-management strategies. (c, d) identity

control

timeline

control

identity

control

control

control

identity

control

illness

coherence 
Figures

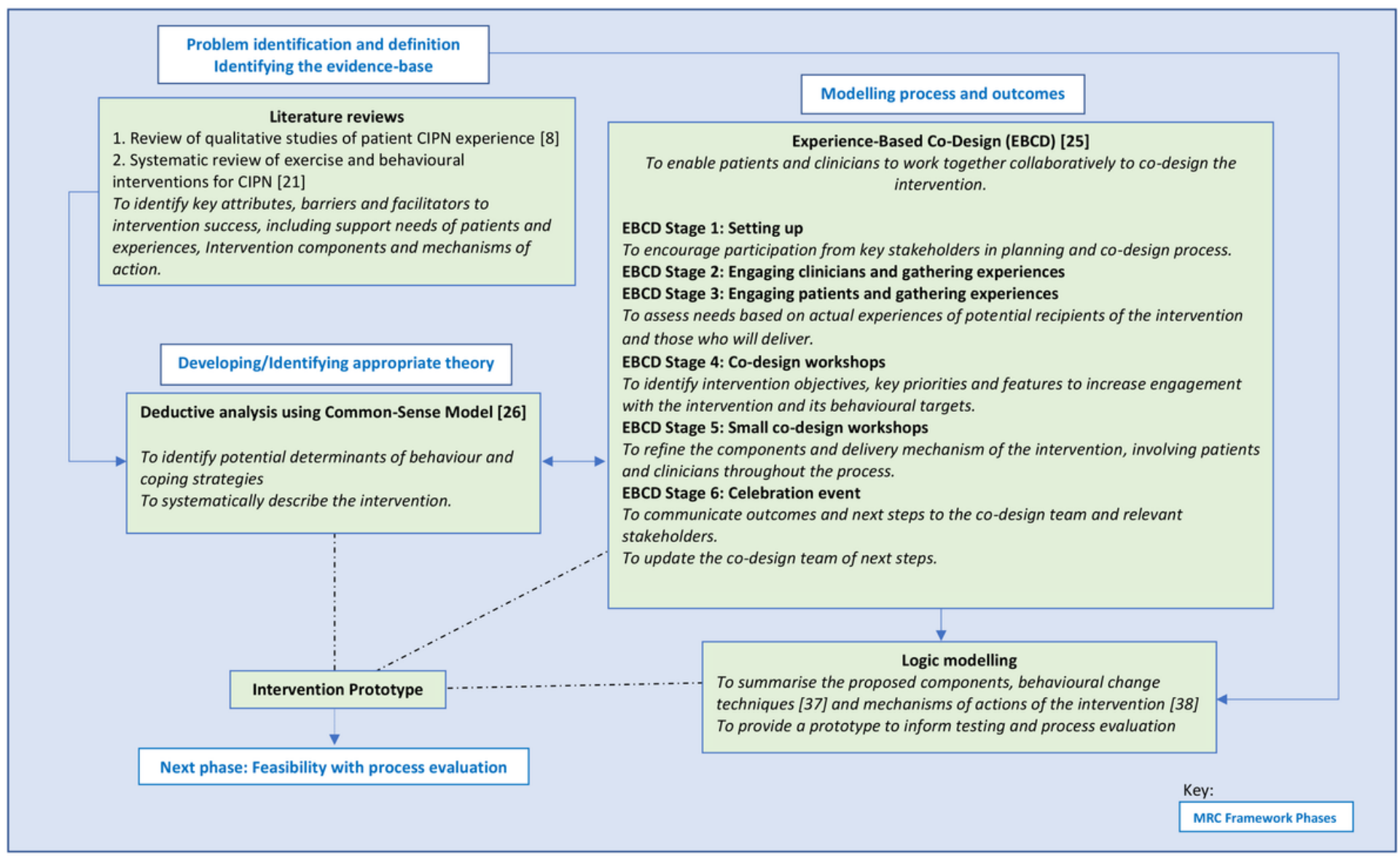

\section{Figure 1}

Key elements of the intervention development as aligned with the MRC Framework for developing complex interventions [1] 


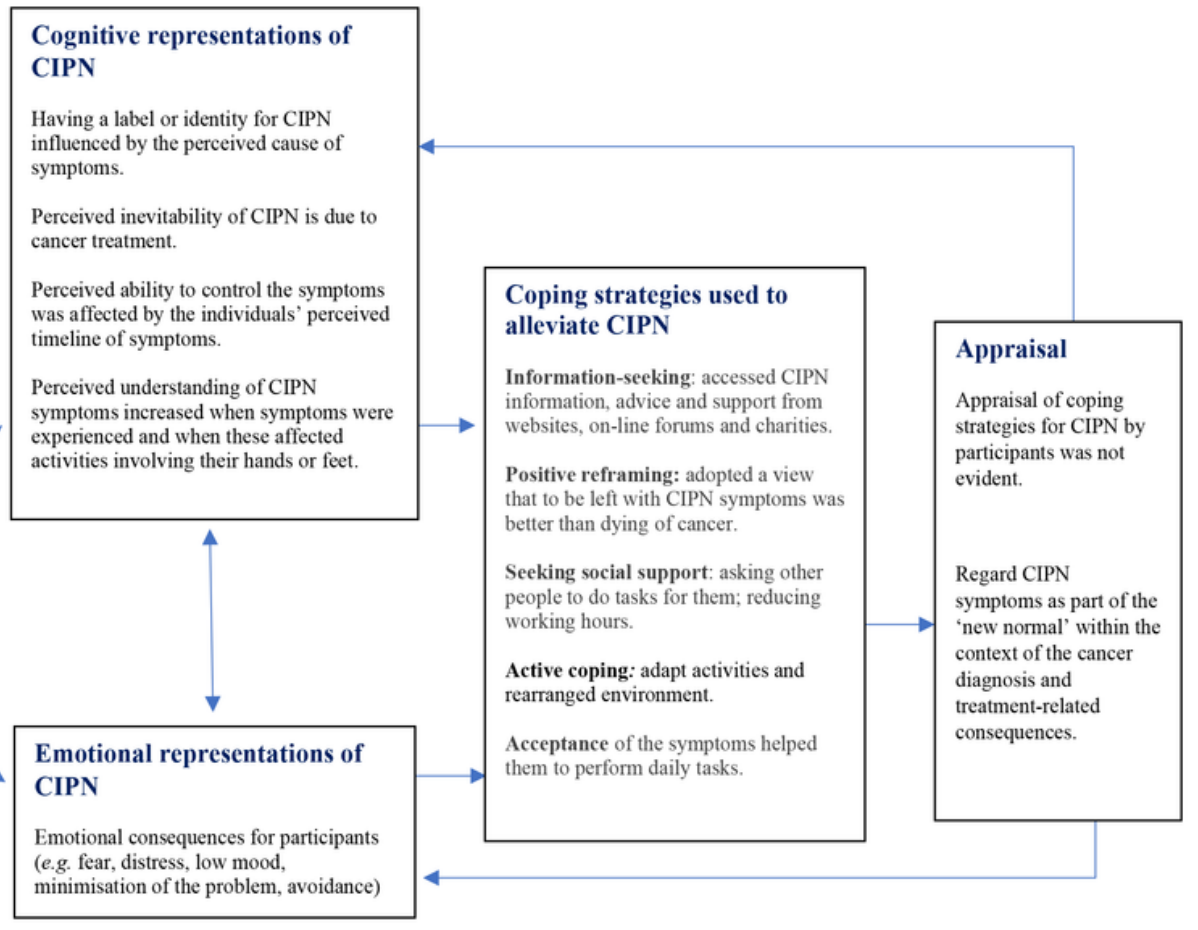

\section{Figure 2}

\section{Self-regulation model of CIPN}

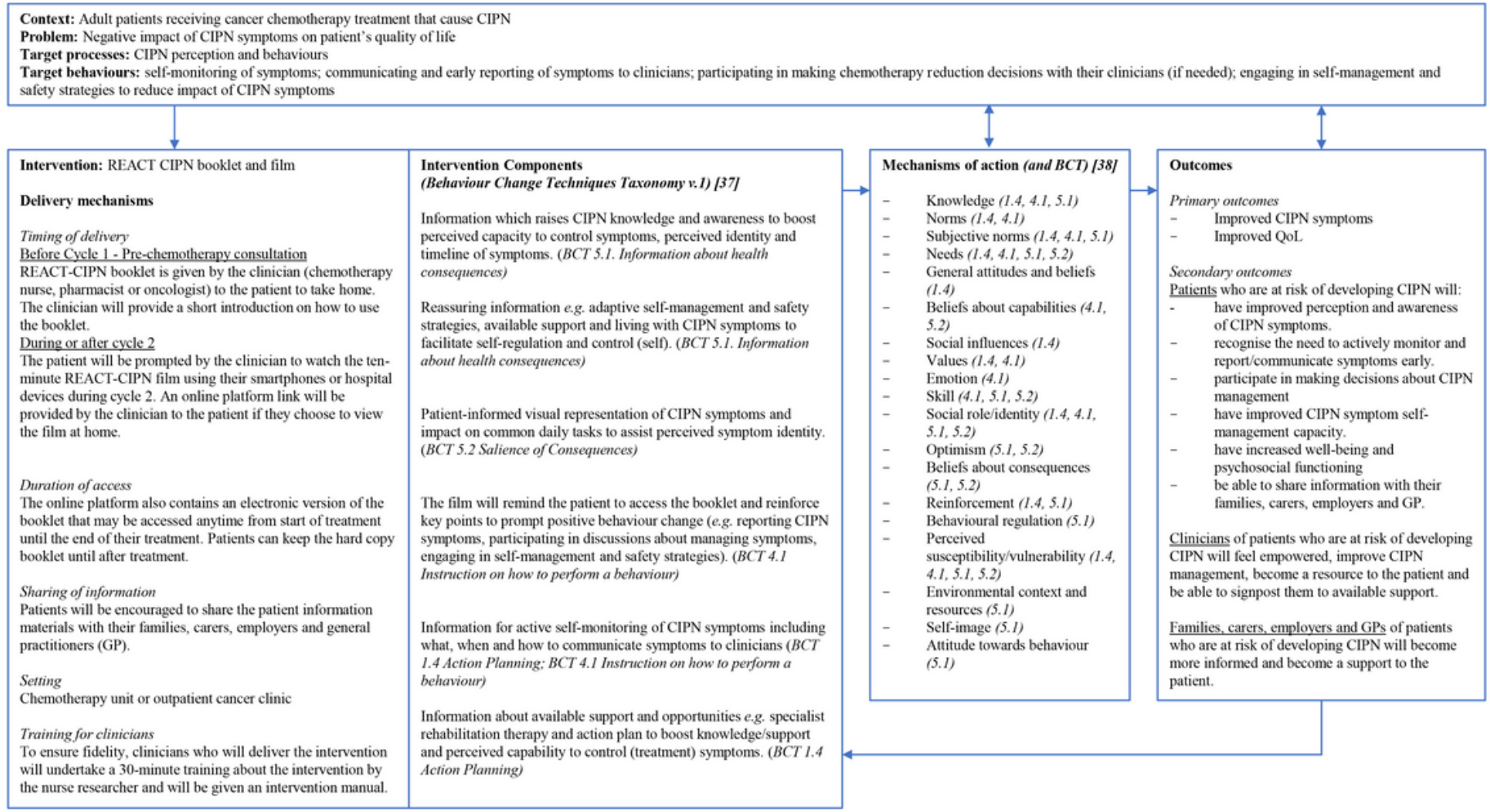

\section{Figure 3}


Logic model of REACT-CIPN intervention components, delivery mechanisms, mechanisms of action and outcomes

\section{Supplementary Files}

This is a list of supplementary files associated with this preprint. Click to download.

- FINAL20210826JSCCCIPNSupplement.pdf 